\title{
Equine influenza
}

\section{Paula J. Spokes ${ }^{\mathrm{A}}$, Ian Roth and Paul K. Armstrong $\mathrm{C}$}

A NSW Public Health Officer Training Program, NSW Department of Health

${ }^{\mathrm{B}} N S W$ Department of Primary Industries

${ }^{\mathrm{C}}$ Biopreparedness Unit, NSW Department of Health

Equine influenza (EI) is an acute viral respiratory disease of horses and other equine species such as donkeys and mules. Symptoms generally include elevated temperature, a deep dry cough and nasal discharge. ${ }^{1,2} \mathrm{EI}$ is highly infectious and serious outbreaks associated with the importation of horses with subclinical infection from endemic areas have occurred in South Africa, Asia and Europe in recent years. ${ }^{3,4}$ The virus is usually transmitted by respiratory secretion droplets through the cough of an infected horse and can survive on skin, fabrics and surfaces of contaminated equipment for up to 24 hours. ${ }^{5}$ Further spread by direct transmission from humans and fomites can subsequently be significant. ${ }^{5}$

Prior to 2007, there were no reported cases of EI in Australia and vaccination against the disease was not practised other than to meet export requirements. Following reports of suspected infection at the Eastern Creek Quarantine Station in New South Wales (NSW), the first reports of EI disease in Australia were made to the NSW Department of Primary Industries by a veterinarian who observed sick horses in Sydney on 24 August 2007. Laboratory testing confirmed that these horses were infected with EI. A statewide lockdown of the movement of horses was declared on 25 August by the NSW Chief Veterinary Officer and the Australian Veterinary Emergency Plan (AUSVETPLAN) equine influenza strategy for disease control was activated. ${ }^{5}$ At the peak of this outbreak, 47000 infected horses on 5943 properties were reported in NSW and, as a result, horse owners across Australia experienced significant economic and social impacts.

Measures taken to control and eradicate the disease included: quarantine and controlled movement of horses; establishment of disease control zones; implementation of decontamination procedures for properties; introduction of surveillance systems to determine the extent of infection, vaccination and awareness; and information campaigns for industry and the wider community. The response to EI involved over 2000 people deployed across NSW as part of a whole-of-government approach. ${ }^{6}$ The response was ultimately successful with Australia declaring freedom from EI on 30 June 2008.

\section{NSW Health involvement}

Epidemiologists, public health nurses, surveillance officers and trainee public health officers from the NSW Department of Health and area health services provided technical advice and assistance to the Department of Primary Industries from September to December 2007. During this time, a number of disease cluster investigations were initiated to determine the mode of transmission for cases of EI that had no apparent epidemiological links, and to study the role of fomite and other modes of virus transmission. One such study, conducted in October 2007, concluded that direct transmission of EI virus via bird plumage or other animals was possible. ${ }^{7}$ Further research would be necessary to confirm the possibility of this transmission mechanism.

\section{Influenza: horses and humans}

While there are similarities between horse and human influenza outbreak management strategies, there are also significant differences in strategies used to control the spread of the diseases.

The Biopreparedness Unit, NSW Department of Health, is responsible for developing procedures for the management of large scale human disease outbreaks in NSW, focussing on pandemic influenza planning. The response for pandemic influenza in NSW aims to contain the spread of infection until a vaccine becomes available. The stock standstill strategy, effective in the containment of EI, would be extremely difficult to replicate for the control of a human influenza outbreak. The objective of such a strategy is to eliminate the transmission outside of the restricted zone, thereby allowing the infection to 'burn itself out'. Similar, but less severe, strategies for humans in an influenza pandemic include isolation, quarantine, social distancing and movement restrictions. More severe restrictions such as isolation of communities or regions would have unacceptably high social and financial costs.

NSW Health involvement in the response to the EI emergency was invaluable in providing insight into the potential challenges of a large-scale human disease outbreak such as pandemic influenza.

This example of a large-scale disease outbreak response highlights the importance of planning for the success of disease control strategies. Important considerations for future pandemic planning include an understanding of the mechanisms for disease transmission and the resource 
and personnel capacity required to respond to a statewide outbreak.

\section{References}

1. Huntingdon PJ. Equine influenza: the disease and its control. Technical report series No. 184. Melbourne: Department of Agriculture and Rural Affairs, Victoria; 1990.

2. Miller WC. Equine influenza: further observations on the "coughing" outbreak, 1965. Vet Rec 1965; 77(16): 455-6.

3. Dalglish AR. The International Movement of Horses: The current infectious disease situation. Proceedings of the 9th International Conference of Racing Analysts and Veterinarians. New Orleans, USA, 1992.

4. Guthrie AJ, Stevens KB, Bosman PP. The circumstances surrounding the outbreak and spread of equine influenza in South Africa. Rev Sci Tech 1999; 18(1): 179-85.
5. Animal Health Australia. Disease strategy: Equine influenza (Version 3.0). Australian Veterinary Emergency Plan (AUSVETPLAN), Edition 3. Canberra: Primary Industries Ministerial Council; 2007.

6. NSW Department of Primary Industries. Summary of the 2007/08 Equine Influenza Outbreak. Sydney: NSW Department of Primary Industries; 2008. Available from: http://www.dpi.nsw.gov.au/agriculture/livestock/horses/health/ general/influenza/summary-of-the-200708-ei-outbreak (Cited 20 March 2009.)

7. Spokes P, Marich A, Musto J, Ward K, Craig A, McAnulty J. Investigation of equine influenza transmission in NSW: walk, wind or wing? N S W Public Health Bull 2009; In press. 